\title{
Survey of mobile ad hoc networks (MANETS)
}

\author{
Abdulghani Saif Kasem Mohammed*, khalid Hamid Bilal \\ Sudan Academy of Sciences, electronic Engineering Department, \\ Khartoum, Sudan
}

\begin{abstract}
:
Mobile Ad Hoc Network (MANET) is the one of the type of ad hoc network, the MANET is a collection of two or more devices or nodes or terminals with wireless communications and networking capability that communicate with each other without the aid of any centralized administrator also the wireless nodes that can dynamically form a network to exchange information without using any existing fixed network infrastructure. And it's an autonomous system in which mobile hosts connected by wireless links are free to be dynamically and sometime act as routers at the same time, In MANET, the mobile nodes require to forward packets for each other to enable communication among nodes outside of transmission range. The nodes in the network are free to move independently in any direction, leave and join the network arbitrarily. Thus a node experiences changes in its link states regularly with other devices. Eventually, the mobility in the ad hoc network, change of link states and other properties of wireless transmission such as attenuation, multipath propagation, interference etc. This paper discuses study of Mobile ad-hoc Networks (MANET): classification, characteristics, structure of MANET and challenges that are imposed by Mobile ad-hoc Networks.
\end{abstract}

KEYWORDS—mobile ad-hoc networks (mantes), VANET, application, challenges

\section{Introduction}

With the widespread rapid development of computers and the wireless communication, the mobile computing has already become the field of computer communications in high-profile link. Mobile Ad Hoc Network (MANET) is a completely wireless connectivity through the nodes constructed by the actions of the network, which usually has a dynamic shape and a limited bandwidth and other features[19], network members may be inside the laptop, Personal Digital Assistant (PDA), mobile phones, MP3 players, and digital cameras and so on as shown in Figure 1. On the Internet, the original Mobility is the term used to denote actions hosts roaming in a different domain; they can retain their own fixed IP address, without the need to constantly changing, which is Mobile IP technology. Mobile IP nodes in the main action is to deal with IP address management, by Home Agent and Foreign Agent to the Mobile Node to packet Tunneling, the Routing and fixed networks are no different from the original; however, Ad Hoc Network to be provided by Mobility is a fully wireless, can be any mobile network infrastructure, without a base station, all the nodes can be any link, each node at the same time take Router work with the Mobile IP completely different levels of Mobility[21]. Early use of the military on the Mobile Packet Radio Networked in fact can be considered the predecessor of MANET, with the IC technology advances, when the high-tech communication equipment, the size, weight continuously decreases, power consumption is getting low, Personal Communication System (Personal Communication System, PCS) concept evolved, from the past few years the rapid popularization of mobile phones can be seen to communicate with others anytime, anywhere, get the latest information, or exchange the required information is no longer a dream, And we have gradually become an integral part of life[20]. Military purposes, as is often considerable danger in field environment, some of the major basic communication facilities, such as base stations, may not be available, in this case, different units, or if you want to communicate between the forces, we must rely on This cannot MANET network infrastructure limitations[23]. In emergency relief, the mountain search and rescue operations at sea, or even have any infrastructure cannot be expected to comply with the topographical constraints and the pressure of time under the pressure, Ad Hoc Network completely wireless and can be any mobile feature is especially suited to disaster relief operations. When personal communication devices and more powerful, some 
assembly occasions, if you need to exchange large amounts of data, whether the transmission of computer files or applications that display. If we can link into a temporary network structure, then the data transmission will be more efficient without the need for large-scale projection equipment would not have point to point link equipment (such as network line or transmission line). The current wireless LAN technology, Bluetooth is has attracted considerable attention as a development plan. Bluetooth's goal is to enable wireless devices to contact with each other, if the adding the design of Ad Hoc Network (MANET)[22].

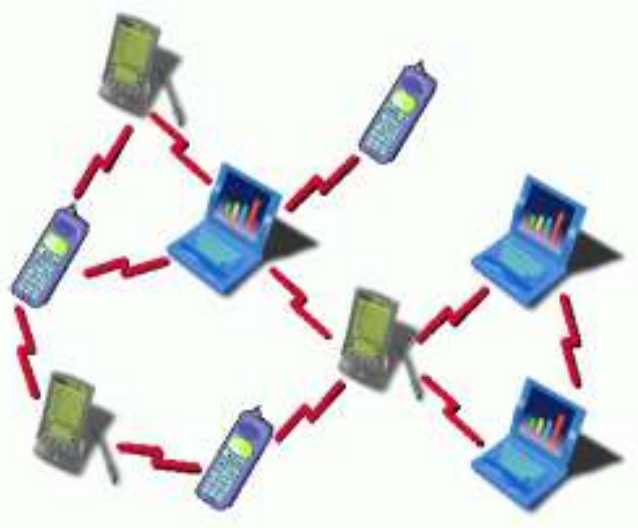

Figure1: MANET

\section{CLASSIFICATION}

TYPES OF MOBILE AD-HOC NETWORKS (MANET'S) ARE [25, 26, 27] :

$>$ Vehicular Ad hoc Networks (VANETs): These are used for communication among Vehicles and between vehicles and roadside equipments.

$>$ Internet based mobile ad hoc networks (iMANET):- These are ad hoc networks that link mobile nodes and fixed Internet-gateway nodes. In such type of networks normal Ad hoc routing algorithms don't apply directly.

$>$ Intelligent vehicular ad hoc networks (InVANETs):- These are a kind of artificial Intelligence that helps vehicles to behave in intelligent manners during vehicle-toVehicle collisions, accidents, drunken driving etc.

\section{MANETS APPLICATIONS}

THE MOST IMPORTANT APPLICATIONS OF MANETs[1, 28-30]:

$>$ Business : One of many possible uses of mobile Ad hoc networks is in some business environments, where the need for collaborative computing might be more important outside the office environment than inside, such as in a business meeting outside the office to brief clients on a given assignment. Fig 2 illustrates the client-server model.

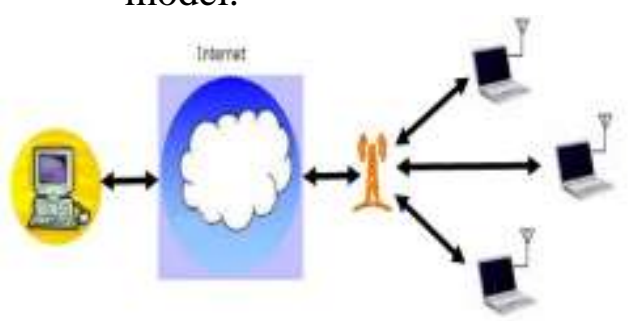

Fig 2: Client-Server Model

$>$ Military:Military applications have motivated early research on Ad hoc networks. The ability to quickly set up a network among military units in hostile territory without any infrastructure support can provide friendly forces with a considerable tactical advantage on the battlefield. For instance, each soldier can carry a mobile device that represents one of the mobile nodes in an Ad hoc network linking all soldiers, tanks, and other vehicles as shown in Fig. 3 Recent advances in robotics have also motivated the idea of automated battlefields in which unmanned fighting vehicles are sent into battle. Supporting military applications requires self-organizing mechanisms that provide robust and reliable communication in dynamic battle situations

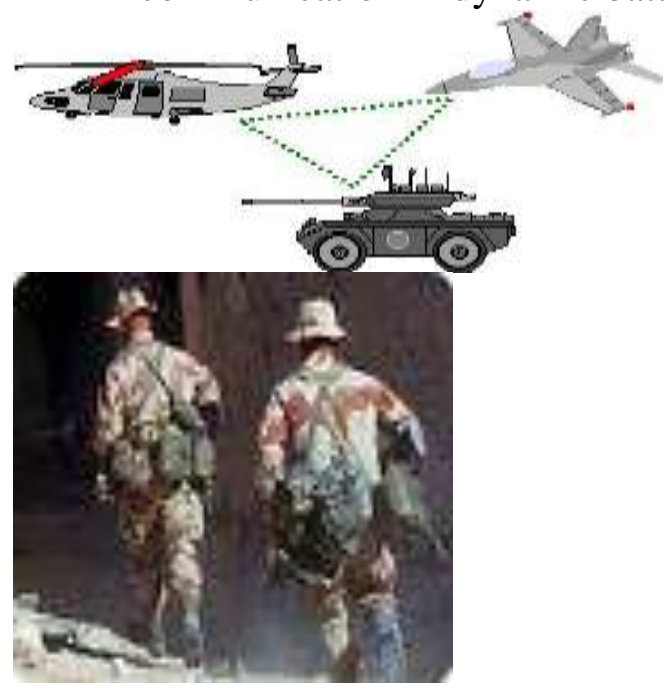

Fig. 3: Soldiers, Tanks and other Vehicles carrying Mobile Devices

$>$ Emergency Operations : Another promising application area for mobile Ad hoc networks is emergency services, including search and rescue and disaster recovery operations. As an example of search and rescue, consider an airline that attaches small wireless devices to the life jackets under each seat. Suppose that the plane has mechanical problems and has to make an emergency landing in the water. Once search and rescue teams arrive at the landing site, they are provided with detailed information about the location (the 
coordinates and potentially the depth) of the victims through the transponders. As a result, the rescue teams can more effectively locate and reach the victims. The mobile devices could also monitor the vital signs of victims, such as heart rate or breathing rate, to prioritize the rescue of victims that are still alive. A similar application arises when disasters, such as earthquakes, blackouts, or bombings occur. The disaster may destroy existing communication infrastructure, preventing critical contact among emergency workers. The emergency response teams can set up Ad hoc networks quickly to replace the destroyed infrastructure.

Home, Office, and Educational: mobile Ad hoc networks also have applications in home and office environments. The simplest and most direct application of Ad hoc networks in both homes and offices is the networking of laptops, PDAs and other WLAN-enabled devices in the absence of a wireless base station. Another home application that falls within the Personal Area Network (PAN) class is wire replacement through wireless links, as in Bluetooth. All periphery devices can connect to a computer through wireless Bluetooth links, a wireless base station. Another home application that falls within the Personal Area Network (PAN) class is wire replacement through wireless links, as in Bluetooth. Ad hoc networks can also enable streaming of video and audio among wireless nodes in the absence of any base station.. Educational and recreational activities can also benefit from Ad hoc networks. For example, students attending a classroom can use their laptops to obtain the latest class material from a professor's laptop as the class progresses. Universities and campus settings, virtual classrooms, Ad hoc communications during meetings or lectures are some of the educational applications of Ad hoc networks. On the recreational side, the mobility and nomadic nature of Ad hoc networks enables richer multi-user games that can incorporate user mobility and proximity into the virtual game environment.

$>$ A Vehicular Ad hoc Network [VANET]: VANETs (Vehicular Ad Hoc Networks) are a new kind of network useful for offering traffic information interchange in a collaborative way between vehicles. They are foreseen to be a great revolution in the driving, providing new services such as: $\star$ Road safety: improve security in dangerous or unexpected driving situations (fog, accident etc. ) incorporating active mechanisms of accidents prevention.

$\star$ Traffic management: dynamic route pacification through collaborative information interchanged between vehicles. Road capacity increment. Trip duration reduction.

$\star$ Cost reduction in the vehicle security incorporation: since the system operation has hardly maintenance, the costs reduction may contribute to spread its deployment.

$\star$ Public transport: increase their efficiency, which attracts users.

Applications based on VANETs are in expansion today. Traffic View is a (Car to Car) application for real time alarms and route planning. Vehicles spread useful traffic information using wireless short range links by using other vehicles as routers, as showing Figure. 4. This way, it is possible to help drivers with information about road status or accidents, to ask a taxi from a mobile device (phone, PDA) or to adapt traffic lights depending on traffic density. Car to Car Consortium $(\mathrm{C} 2 \mathrm{C})$ is a non lucrative organization created by European vehicles manufacturers (Audi, BMW, Daimler Chrysler, Fiat, Renault and Volkswagen). Its main objective is to create a European open standard for the C2C communication systems and Car to Infrastructure (C2I) to achieve communications between infrastructure and vehicles. Also the assignation of an exclusive band frequency is promoted as well as the creation of a world level communications standard between vehicles. In VANETs, the relative speed between nodes is much larger, so link life is lower than in MANETs. To avoid the odd effects due to its lower link life, routing protocols have to be fast and efficient. There is no need of fixed infrastructure, and only some Internet gateways are needed along the road to access the network, if required. It is a very scalable network, cheap and easy to arrive to much kind of different users. Some characteristics of VANETs compared to infrastructure based and cellular communications are shown in Figure.4 VANETs can offer a wider type of services to contribute to a safe driving, being its low cost of fundamental relevance. The European Commission is a strong supporter of this technology capable to prevent accidents using intelligent vehicle safety systems, such as airbags, seatbelts, ABS brakes etc. along with VANETs which can further improve safety at a low cost. For instance, safety Aware seeks to accelerate the market introduction of this kind of life-saving technologies. 


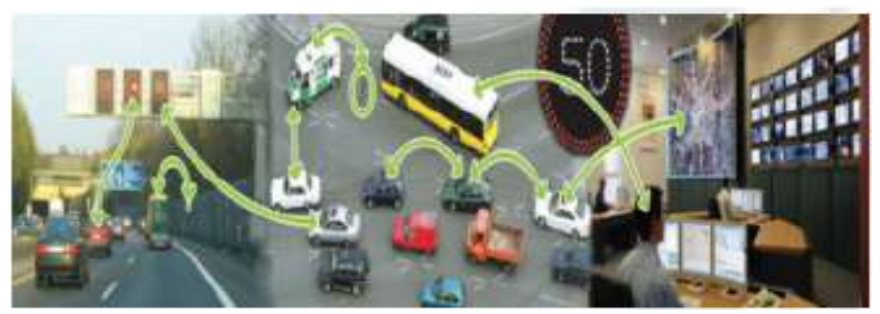

Figure 4: VANETs use traffic information from central bases.

Table1: Main differences between MANETs and VANETs.

\begin{tabular}{|l|l}
\hline \multicolumn{1}{|c|}{ MANETs } & VANETs \\
\hline - No infrastructure & - Network nodes are vehicle w \\
- Random movement & - Nodes move following guidel \\
- Limited battery life & - No energy limitations \\
\hline
\end{tabular}

$>$ Wireless Sensor Networks : Advances in processor, memory and radio technology will enable small and cheap nodes capable of sensing, communication and computation. Networks of such nodes called wireless sensor networks can coordinate to perform distributed sensing of environmental phenomena.

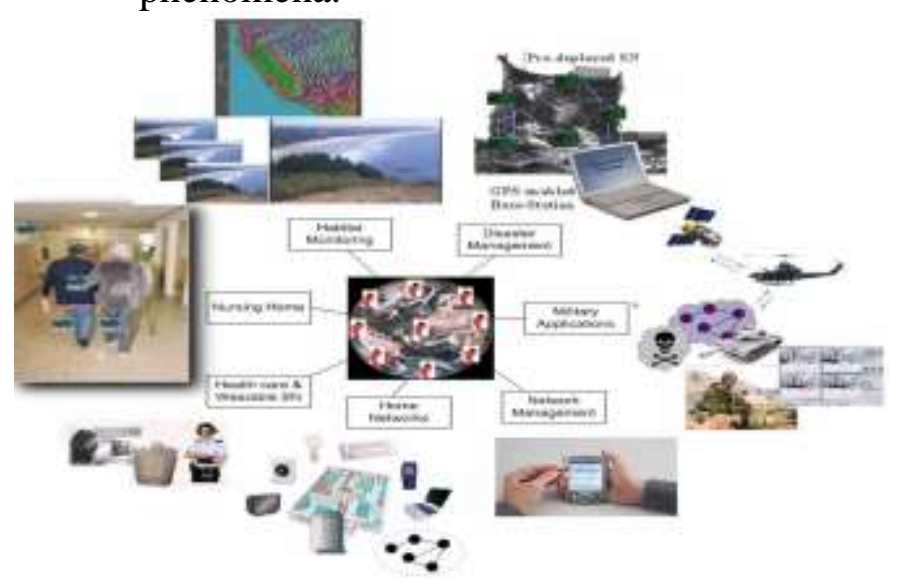

Fig. 5: Applications of Sensor Networks

A sensor network is a network of many tiny disposable low power devices, called nodes, which are spatially distributed in order to perform an application-oriented global task. These nodes form a network by communicating with each other either directly or through other nodes. One or more nodes among them will serve as sink(s) that are capable of communicating with the user either directly or through the existing wired networks. The primary component of the network is the sensor, essential for monitoring real world physical conditions such as sound, temperature, humidity, intensity, vibration, pressure, motion, pollutants etc. at different locations. Wireless sensor networks can be considered as special case of mobile Ad hoc networks (MANET) with reduced or no mobility as shown in Fig .5.

\section{CHARACTERISTICS OF MANET}

The most important characteristics of MANET are
$[11,26]:$

$>$ Cooperation: If the source node and destination node are out of range with each other then the communication between them takes place with the cooperation of other nodes such that a valid and optimum chain of mutually connected nodes is formed. This is also called as multi-hop communication. In multi hop communication each node is to act as a host as well as router simultaneously.

Dynamism of Topology: The nodes of MANET are randomly, frequently and unpredictably mobile within the network. These nodes may leave or join the network at any point of time, thereby significantly affecting the status of trust among nodes and the complexity of routing. Such mobility entails that topology of the network as well as the connectivity between the hosts is unpredictable. so the management of the network environment is a function of the participating nodes.

$>$ Lack of fixed infrastructure: The absence of a fixed or control infrastructure is a key feature of MANETs. This eliminates the possibility to establish a centralized authority to control the network characteristics. Due to this absence of authority, traditional techniques of network management and security are scarcely applicable to MANETs.

Resource Constraints: MANETs are a set of mobile devices which are of lower limited power capacity, computational capacity, memory, bandwidth etc. So in order to achieve a secure and reliable communication between nodes, these resource constraints make the task more enduring all of the routing protocols in MANETs depends on active cooperation of nodes to provide routing between the nodes and to establish and operate the Network.

> scalability:Currently, popular network management algorithms were mostly designed to work on fixed or relatively small wireless networks. Many mobile ad hoc network applications involve large networks with tens of thousands of nodes, as found for example, in sensor networks and tactical networks. Scalability is critical to the successful deployment of these networks. The steps toward a large network consisting of nodes with limited resources are not straightforward, and present many challenges that are still to be solved in areas such as: addressing, routing, location management, configuration management, interoperability, 
security, high capacity wireless technologies, etc.

Autonomous and infrastructure-less: MANET does not rely on any established infrastructure or centralized administration. Each node operates in distributed peer-to-peer mode, acts as an independent router and generates independent data. Network management has to be distributed across different nodes, which brings difficulty in fault detection and management.

\section{MANET ARCHITECTURE:}

The architecture of Mobile Ad-hoc Network (MANET) is shown in figure 6. The network architecture is grouped into main three categories:

- Enabling technologies - Networking

Middleware and applications

Enabling Technology: Depending on their coverage area, these technologies are classified into several classes:

$>$ Body (BAN), Personal (PAN), Local (LAN), Metropolitan (MAN) and Wide area networks (WAN) [7, 8].

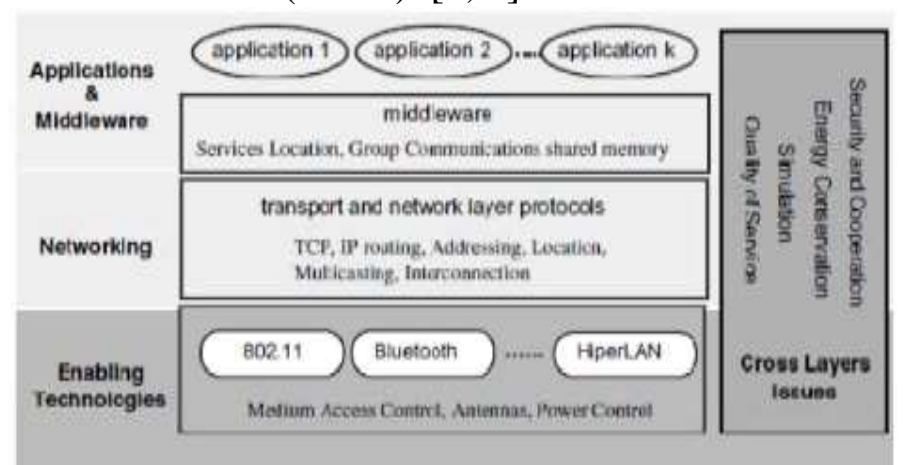

Fig. 6 : A simple MANET architecture

A body area network (BAN) is strongly connected with wearable computers. A wearable computer distributes on the body its components like as head mounted displays, microphones, earphones, etc. and the BAN provides the connectivity among these devices. With respect to the human body range, $t$ he communicating range of a BAN is $1-2 \mathrm{~m}$ [9]. The Personal area networks (PAN) connect the mobile devices which are carried by users to other mobile and stationary devices[11]. A PAN communicating range is typically up to $10 \mathrm{~m}$. Wireless LANs (WLANs) support 100-500 m communication range for a single building, or a cluster of buildings Wideand Metropolitan-area (WAN-MAN) ad hoc networks are mobile multi-hop wireless networks that face many challenges which are still to be solved (e.g., addressing, routing, location management, security, etc.), and their availability is not on immediate horizon[10].

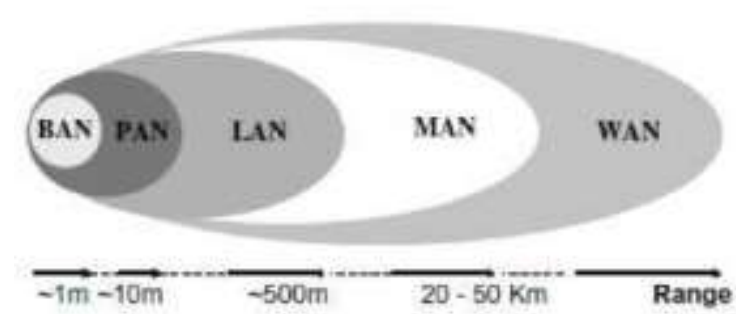

Fig 7 : Ad hoc networks Taxonomy

Networking[12, 13, 14]: In MANET, most of the main functionalities of the Networking protocols need to be re-designed for the self organizing, dynamic, volatile, peer-to-peer communication environment. The main target of networking protocols is to use the one-hop transmission services which are provided by the enabling technologies to construct end-toend (reliable) delivery services, from a sender to one (or more) receiver(s). In case of establish an end-to-end communication, the sender needs to locate the receiver within the network.

Middleware \& Applications[18, 17, 15, 16]: The introduction of new technologies like as the WiFi, Bluetooth, IEEE 802.11, WiMAX and Hyper LAN greatly facilitates the deployment of ad hoc technology, and new ad hoc networking applications appeared mainly in specialized fields such as emergency services, disaster recovery and environment monitoring. In addition, MANET flexibility makes this technology attractive for several application, for example, in personal area networking, home networking,, search andrescue operations, commercial, educational applications, and sensor networks. Mobile ad hoc systems currently developed adopt the approach of not having a middleware, but rather rely on each application to handle all the services it needs.

\section{Advantages of MANET}

The main advantages of MANET are [24]:

$\square$ They provide access to information and services regardless of geographic position.

$\square$ These networks can be set up at any place and time.

$\square$ Independence from central network administration. Self-configuring network, nodes are also act as routers. Less expensive as compared to wired network.

$\square$ Scalable - accommodates the addition of more nodes.

$\square$ Improved flexibility.

$\square$ They are robust due to decentralize administration. 
7.Challenges In Mobile Ad-Hoc Networks:

The most important challenges of the MANET are [1, 2-6]:

Routing: Routing is a significant point of view with researchers since routing protocols is essential issue in this field, because changes in network topology occur frequently. An efficient and intelligent routing protocol is required to cope with highly dynamic and fluid network conditions

Route Changes Due To Mobility: The network topology in an ad hoc wireless network is highly dynamic due to the movement of nodes.

Limited Bandwidth: Wireless link continue to have significantly lower capacity than infrastructure networks. In addition, the realized throughput of wireless communication after accounting for the effect of multiple access, fading, noise, and interference conditions, etc., is often much less than a radio's maximum transmission rate.

Dynamic Topology : Dynamic topology membership may disturb the trust relationship among nodes.

Battery Constraints: Devices used in these networks have restrictions on the power source in order to maintain portability, size and weight of the device.

Radio Interface: Mobile nodes rely on the radio interface or antenna to transmit packets. Packet forwarding or receiving via radio interface or antenna techniques in MANETs are useful investigations.

Power Management: A power management approach would help reducing power consumption and hence prolonging the battery life of mobile nodes. Because most devices operate on batteries, power management becomes an important issue.

Security Threats : The wireless mobile ad hoc nature of MANETs brings new security challenges to the network design. As the wireless medium is vulnerable to eavesdropping and ad hoc network functionality is established through node cooperation, mobile ad hoc networks are intrinsically exposed to numerous security attacks.

QOS: Quality of Service (QoS) and Multimedia require high bandwidth, low delay, and high reliability.

\section{Conclusion}

This paper discussed overview of MANET. In addition we focus on the most important characteristics and challenges. Also this paper provided the type of MANETS and different applications of MANET. The manet is infrastructureless network(wireless communication) which send the data from source to destination without establishing any connection of wires, means the source node and destination is known but path of transmission is not specified, each time the different route is Selected. MANET device is free for move anywhere in any direction and goal is each device maintains information required to properly route traffic. MANET devices can be operating independently or otherwise connected to the internet. - The brief discussion in this paper shows that, despite the large efforts of the MANET research community and the rapid progress .

\section{References}

[1] K. S. Ali and U. Kulkarni, "Characteristics, Applications and Challenges in Mobile AdHoc Networks (MANET): Overview," Wireless Networks, vol. 3, 2015.

[2] O. Bang and P. L. Ramteke, "MANET: history, challenges and applications," International Journal of Application or Innovation in Engineering \& Management (IJAIEM), vol. 2, pp. 249-251, 2013.

[3] K. BR, L. C. Reddy, and P. S. Hiremath, "Mobile Ad Hoc Networks: Issues, Research Trends And Experiments," 2008.

[4] M. L. Raja and C. D. S. S. Baboo, "An Overview of MANET: Applications, Attacks and Challenges," International Journal of Computer Science and Mobile Computing (IJCSMC), vol. 3, pp. 408 - 417, 2014.

[5] S. J. Shivi Sharma, "Mobile Ad Hoc Network: Issues, Research Trend and Challenges," International Journal of Advanced Research in Computer Science and Software Engineering, vol. 5, pp. 1625 1630, 2015.

[6] T. Qiu, N. Chen, K. Li, D. Qiao, and Z. Fu, "Heterogeneous ad hoc networks: Architectures, advances and challenges," Ad Hoc Networks, vol. 55, pp. 143-152, 2017.

[7] M. Conti, Body, personal, and local wireless ad hoc networks, in: M. Ilyas (Ed.), Handbook of Ad Hoc Networks, CRC Press, New York, 2003 (Chapter 1).

[8] M.S. Corson, J.P. Maker, J.H. Cernicione, Internet-based mobile ad hoc networking, IEEE Internet Computing 3 (4) (1999) 63-70.

[9] W. Stallings, Local \& Metropolitan Area Networks, Prentice Hall, Englewood Cliffs, NJ, 1996.

[10] Web site of the IEEE 802.11 WLAN: http://grouper.

ieee.org/grups/802/11/main.html.

[11] Web site of the Bluetooth Special Interest Group: http:// www.bluetooth.com/. 
[12] Y. Bing Lin, I. Chlamtac, Wireless and Mobile Network Architectures, Wiley, New York, 2000.

[13] Martin Mauve, Jorg Widmer, Hannes Hartenstein, A survey on position-based routing in mobile ad-hoc networks, IEEE Network 15 (6) (2001) 30-39. [14] S. Basagni, I. Chlamtac, V. Syrotiuk, B. Woodward, A distance routing effect algorithm for mobility (DREAM), in: Proceedings of The Fourth Annual ACM/IEEE International Conference on Mobile Computing and Networking (MOBICOM _98), Dallas, TX, USA, October 25-30, 1998.

[14] S. Dhar, "MANET: Applications, Issues, and Challenges for the Future," International Journal of Business Data Communications and Networking (IJBDCN), vol. 1, pp. 66-92, 2005.

[15] A.J. Goldsmith, S.B. Wicker, Design challenges for energy-constrained ad hoc wireless networks, IEEE Wireless Communications 9 (4) (2002) 8-27.

[16] Cecilia Mascolo, Licia Capra, Wolfgang Emmerich, Middleware for mobile computing (a survey), in: Enrico Gregori, Giuseppe Anastasi, Stefano Basagni (Eds.),

Advanced Lectures on Networking, Lecture Notes in Computer Science, vol. 2497, Springer, Berlin, 2002.

[17] Special issue "Special feature on middleware for mobile \& pervasive", ACM Mobile Computing and Communications Review 6 (4) (October 2002).

[18] E. Anceaume, A.K. Datta, M. Gradinariu, G. Simon, Publish/subscribe scheme for mobile networks, in: Proceedings of the ACM Workshop on Principles of Mobile

Computing 2002, pp. 74-81.

[19] Bimal H Patel, Parth D Shah, Harikrishna B Jethva, Nishidh Chavda, "Issues and Imperatives of Ad hoc Networks", International Journal of Computer Applications (0975 - 8887) Volume 62 No.13, January 2013

[20] I. J. Adnane, A., David, S. and Parrein, B, "Multipath optimized link state routing for mobile ad hoc networks", Ad hoc Networks 9, pp-28-47, 2011.

[21] P. Rama Devi and Dr. D. Srinivasa Rao," Congestion Adaptive Hybrid Multi-path Routing Protocol for Load Balancing in Mobile Ad hoc Networks", International Journal of Computer Science and
Telecommunications ,Volume 3, Issue 12, December 2012.

[22] Lokesh Malviya, Akhilesh A. Waoo, Sanjay Sharma, "New Load Balanced Multi-Path Dynamic Source Routing Protocol for Mobile Ad hoc Network", International Journal of Computer Applications (0975 - 8887) Volume 61 - No.19, January 2013.

[23] www.techoprdia.com/defination/14527/wirele ssssensor- network-wmn, "Wireless sensor network".

[24] K. Sreenivasulu, E. V. Prasad, A. Subramanyam, "Performance Analysis of MANET Reactive Routing under Security", International Journal of Computer

Applications (0975 - 8887) volume-60 No.7, December 2012.

[25] Pooja Malik, "Consequences of limited manet resources", International Journal of Advanced Research in Computer Science and Software Engineering, Volume 2, Issue 1, January 2012 ISSN: 2277 128X.

[26] K. Sreenivasulu, E. V. Prasad, A. Subramanyam, "Performance Analysis of MANET Reactive Routing under Security", International Journal of Computer Applications (0975 - 8887) volume-60 No.7, December 2012.

[27] Bimal H Patel, Parth D Shah, Harikrishna B Jethva, Nishidh Chavda, "Issues and Imperatives of Ad hoc Networks", International Journal of Computer Applications (0975 - 8887) Volume 62 No.13, January 2013.

[28] C. HERNÁNDEZ and W. EDUARDO, "Quality of service routing and mechanisms for improving video streaming over mobile wireless ad hoc networks," Editorial Universitat Politècnica de València, 2015.

[29] Yih-Chun Hu and David B. Johnson. Caching Strategies in On-Demand Routing Protocols for Wireless Ad Hoc Networks. In Proceedings of the Sixth Annual ACM/IEEE International Conference on Mobile Computing and Networking (MobiCom'00), pages 231-242, Boston, MA USA, August 2000.

[30] P. Arce Vila, "Hierarchical routing and crosslayer mechanisms for improving video streaming quality of service over mobile wireless ad hoc networks," Editorial Universitat Politècnica de València, 2014. 